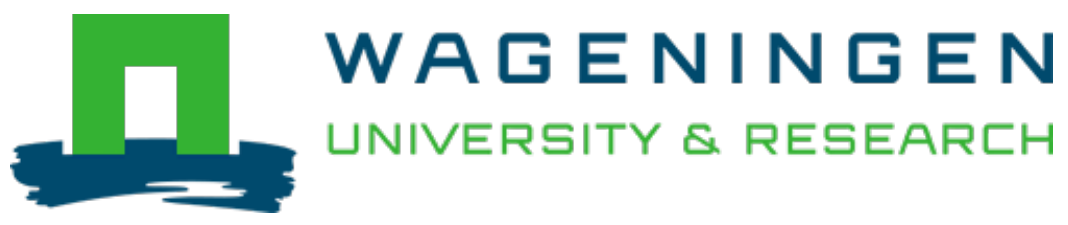

\title{
Minimization of Antinutrients in Idli by Using Response Surface Process Optimization
}

\author{
Journal of Food Processing and Preservation
}

Sharma, Anand; Kumari, Sarita; Nout, Martinus J.R.; Sarkar, Prabir K.

https://doi.org/10.1111/jfpp.13099

This publication is made publicly available in the institutional repository of Wageningen University and Research, under the terms of article $25 \mathrm{fa}$ of the Dutch Copyright Act, also known as the Amendment Taverne. This has been done with explicit consent by the author.

Article 25 fa states that the author of a short scientific work funded either wholly or partially by Dutch public funds is entitled to make that work publicly available for no consideration following a reasonable period of time after the work was first published, provided that clear reference is made to the source of the first publication of the work.

This publication is distributed under The Association of Universities in the Netherlands (VSNU) 'Article $25 \mathrm{fa}$ implementation' project. In this project research outputs of researchers employed by Dutch Universities that comply with the legal requirements of Article $25 \mathrm{fa}$ of the Dutch Copyright Act are distributed online and free of cost or other barriers in institutional repositories. Research outputs are distributed six months after their first online publication in the original published version and with proper attribution to the source of the original publication.

You are permitted to download and use the publication for personal purposes. All rights remain with the author(s) and / or copyright owner(s) of this work. Any use of the publication or parts of it other than authorised under article $25 \mathrm{fa}$ of the Dutch Copyright act is prohibited. Wageningen University \& Research and the author(s) of this publication shall not be held responsible or liable for any damages resulting from your (re)use of this publication.

For questions regarding the public availability of this publication please contact openscience.library@wur.nl 


\title{
MINIMIZATION OF ANTINUTRIENTS IN IDLI BY USING RESPONSE SURFACE PROCESS OPTIMIZATION
}

\author{
ANAND SHARMA ${ }^{1}$, SARITA KUMARI ${ }^{1}$, MARTINUS J.R. NOUT ${ }^{2}$ and PRABIR K. SARKAR ${ }^{1,3}$ \\ ${ }^{1}$ Microbiology Laboratory, Department of Botany, University of North Bengal, Siliguri, 734 013, India \\ ${ }^{2}$ Laboratory of Food Microbiology, Wageningen University, 6700 AA Wageningen, The Netherlands
}

${ }^{3}$ Corresponding author.

TEL: +91-353-2776-337;

FAX: +91-353-2699-001;

EMAIL: pksarkar.nbu@gmail.com

Received for Publication January 3, 2016

Accepted for Publication May 3, 2016

doi:10.1111/jfpp.13099

\begin{abstract}
Deploying response surface methodology, the stages of idli preparation were optimized for minimizing the level of antinutrients. Under optimum conditions of soaking blackgram dal (1:5 of dal and water at $16 \mathrm{C}$, and $\mathrm{pH} 4.0$ for $18 \mathrm{~h}$ ) and rice ( $1: 5$ of rice and water at $16 \mathrm{C}$, and $\mathrm{pH} 5.6$ for $18 \mathrm{~h}$ ), the tannins content, trypsin inhibitor activity and hemagglutinating activity reduced, while phytic acid content remained unchanged. The optimum conditions for fermentation of dal-rice (1:2) mixed batter were $16 \mathrm{~g} / \mathrm{kg}$ common salt supplementation and $19 \mathrm{~h}$ at $35 \mathrm{C}$, resulting in a decrease in all the antinutrient levels, except amines. Steaming for an optimized period of 20 min further reduced the phytic acid content and trypsin inhibitor activity. In idli, while total biogenic amines content increased by $339 \%$ over raw ingredients, tannins content, phytic acid content, trypsin inhibitor activity and hemagglutinating activity decreased by $100,89,58$ and $100 \%$, respectively.
\end{abstract}

\section{PRACTICAL APPLICATIONS}

For idli preparation, the optimization of processing stages using response surface methodology significantly minimized the level of antinutrients from both blackgram dal and rice without affecting the organoleptic attributes of the product. The optimized process parameters can be applied to household level and are also useful in scaling up idli production with a minimum level of antinutrients and better consumer acceptability. The outcome of this research can be exploited to other legume-based foods as well, particularly in developing regions where the consequences of antinutrients may exacerbate malnutrition and disease, thus effectively utilizing full potential of the legumes as human and animal foods.

\section{INTRODUCTION}

In countries, where scarcity of animal protein prevails, legumes play an important role in the human diet. Legumes fulfill the basic nutritional requirements, such as proteins, dietary fibers, unsaturated fatty acids, vitamins and minerals. Legumes, blended with cereals, provide nutritional and functional proteins having a well-balanced essential amino acid profile (Boye et al. 2010). A significant part of the world's human population relies on legumes as staple food for subsistence, particularly in combination with cereals.

However, besides having these beneficial qualities, legumes and cereals contain a considerable amount of antinutrients, such as galacto-oligosaccharides, nonprotein amino acids, tannins, phytates, trypsin inhibitors, hemag- glutins or lectins and biogenic amines which limit their consumption (Hemalatha et al. 2007). Reduction of antinutrients in legumes can be achieved either by selection of plant genotypes with low levels of such factors or through postharvest processing. Since antinutrients are important to the plants as they function as potent defense compounds against herbivores and pathogens, postharvest processing has been the strategy for their elimination from seeds. As many of the antinutrients are toxic, unpalatable and/or indigestible for human consumption, the traditional domestic means of their reduction consists mainly of dehulling, leaching, germination, heating and fermentation. Structure of antinutrients and their chemical properties, especially heat 
lability, dictate which physical process will be more effective in their reduction or removal.

Idli is a classic example of cereal-legume mixture food, consumed throughout India, especially in southern parts, and Sri Lanka (Aidoo et al. 2006). Traditionally, idli is prepared by soaking blackgram (Vigna mungo (L.) Hepper; synonym Phaseolus mungo L.) dal (dehulled split seeds) and white polished rice (Oryza sativa L.) separately. The soaked rice is ground coarsely, while soaked dal is ground to a smooth, viscous paste. The slurry and paste are mixed at different ratios with common salt to form a thick batter that is left overnight at ambient temperature. The leavened batter is poured into the cups of an idli steamer and steamed until starch is gelatinized to prepare soft and spongy cakes having a pleasant acid flavor (Aidoo et al. 2006; Rakshit et al. 2015). Idli is usually eaten with chutney (a batter made of coconut) and sambar (spicy vegetable soup containing tamarind juice). Idli is fermented naturally. Many times use of the same utensils helps to stabilize a mixed microbiota during fermentation. The leavening and flavor formation of idli are achieved by the activities of lactic acid bacteria, such as Leuconostoc mesenteroides, Enterococcus faecalis and Pediococcus dextrinicus, and yeasts such as Saccharomyces cerevisiae, Pichia anomala, Debaryomyces hansenii, Trichosporon pullulans and Trichosporon cutaneum (Nout et al. 2007).

The fate of oligosaccharides in blackgram during its processing for preparing idli has been reported elsewhere (Rakshit et al. 2015). However, reports on the other major antinutrients, such as tannins, phytic acid, total biogenic amines, trypsin inhibitor activity and hemagglutinating activity exhibited varied results under different conditions.

Response surface methodology (RSM) is a powerful tool that is useful for applications in which a response is influenced by several factors (Montgomery 2005). Since RSM also evaluates the interactions between one or more response variables with fewer experimental runs, it is used extensively for optimization of different food processes. Hence, the present study aimed to use RSM for optimization of the traditional domestic processing stages in achieving preparation of idli having a minimum level of antinutrients.

\section{MATERIALS AND METHODS}

\section{Sampling}

Blackgram dal and white polished rice were purchased from a retailer in Siliguri town, packed in an air-tight aluminum container and stored at ambient temperature (22-30C) for earliest use (within 2 weeks).

\section{Experimental Design}

Preliminary experimental trials conducted in the laboratory and literature survey (Rakshit et al. 2015) enabled us to choose the minimum and maximum values of the independent variables. The influence of variables of each stage was evaluated using a central composite rotatable design (CCRD). Experiments were performed in triplicate sets, according to combinations for each processing stage to obtain an optimized condition using Design Expert v. 8.0 (Stat-Ease Inc., Minneapolis, MN). The soaking stage consisted of 30 experimental runs having 16 factorial points, eight axial points and six replicates at central points (Tables 1 and 2). While optimally soaked dal was ground to a smooth paste, optimally soaked rice was coarsely ground. The dal paste and rice slurry were mixed to get a thick batter which was used for optimization of the subsequent (fermentation) stage employing three independent variables. This stage consisted of 20 experimental runs having eight factorial points, six axial points and six replicates at the central points (Table 3). As per the experimental condition, different amounts of common salt (sodium chloride) were added. At different fermentation times, the bottles were sampled for analysis of antinutrients. The results obtained were used to optimize fermentation condition. Optimally fermented idli batter was used in the final stage (steaming), where a singleindependent variable consisted of seven experimental runs (Table 4). After model fitting of each processing stage, 3D response surfaces were generated to decipher the relation between independent and response variables.

\section{Viable Cell Count}

A $10 \mathrm{~g}$ sample was homogenized with $90 \mathrm{~mL}$ sterile peptone-physiological saline $(1 \mathrm{~g} / \mathrm{L}$ neutral peptone, $8.5 \mathrm{~g} / \mathrm{L}$ sodium chloride, $\mathrm{pH}$ 7.2) in a stomacher lab-blender 400 (Seward Medical, London, UK) for $1 \mathrm{~min}$ at "normal" speed. One milliliter of the appropriate dilution was mixed with $15 \mathrm{~mL}$ molten (45C) plate count agar (M091A; HiMedia Laboratories, Mumbai, India) for total aerobic mesophilic bacterial count, MRS agar (HiMedia M641) for lactic acid bacterial count and tryptone glucose yeast extract agar (HiMedia M014) supplemented with $10 \mathrm{IU} / \mathrm{mL}$ benzylpenicillin and $12 \mu \mathrm{g} / \mathrm{mL}$ streptomycin sulfate for yeast count. The plate count agar and tryptone glucose yeast extract agar plates were incubated at $37 \mathrm{C}$ for $24 \mathrm{~h}$, while MRS agar plates were incubated for $48 \mathrm{~h}$ at $30 \mathrm{C}$ in an anaerobic culture jar (HiMedia LE002A).

\section{pH and Titratable Acidity}

For determining $\mathrm{pH}$ and titratable acidity, the methods described in AOAC (1990) were followed. 
TABLE 1. EXPERIMENTAL VALUES, BASED ON RSM DESIGN, OF ANTINUTRIENTS IN SOAKED DAL

\begin{tabular}{|c|c|c|c|c|c|c|c|c|c|c|}
\hline \multirow[b]{2}{*}{ Run } & \multicolumn{4}{|c|}{ Soaking condition (variable) } & \multirow[b]{2}{*}{ Final $\mathrm{pH}$} & \multicolumn{5}{|c|}{ Antinutrient (per g dry wt)* } \\
\hline & $\begin{array}{l}\text { Dal:water } \\
(w / w)\end{array}$ & $t(\mathrm{~h})$ & $T(\mathrm{C})$ & Initial pH & & $\mathrm{TC}(\mathrm{mg})$ & PAC (mg) & $\mathrm{TIA}(\mathrm{U})$ & $\mathrm{HA}(\mathrm{U})$ & $\operatorname{TBAC}(\mu \mathrm{g})$ \\
\hline 1 & $1: 1$ & 12 & 26 & 6.0 & $5.7 \pm 0.3$ & $0.46 \pm 0.09$ & $4.9 \pm 0$ & $111 \pm 2.3$ & $160 \pm 0$ & $296 \pm 1$ \\
\hline 2 & $1: 7$ & 12 & 4 & 6.0 & $5.8 \pm 0.3$ & $0.23 \pm 0.12$ & $4.1 \pm 0.3$ & $81 \pm 0.6$ & $133 \pm 27$ & $305 \pm 2$ \\
\hline 3 & $1: 7$ & 24 & 26 & 6.0 & $5.6 \pm 0$ & $0.19 \pm 0$ & $3.0 \pm 0$ & $65 \pm 0$ & $120 \pm 40$ & $306 \pm 2$ \\
\hline 4 & $1: 10$ & 6 & 37 & 4.0 & $3.6 \pm 0$ & $0.32 \pm 0.03$ & $4.2 \pm 0$ & $85 \pm 1.4$ & $107 \pm 27$ & $324 \pm 2$ \\
\hline 5 & $1: 10$ & 6 & 37 & 8.0 & $6.5 \pm 0$ & $0.32 \pm 0.03$ & $4.2 \pm 0$ & $83 \pm 1.9$ & $133 \pm 27$ & $304 \pm 1$ \\
\hline 6 & $1: 7$ & 12 & 26 & 6.0 & $5.6 \pm 0$ & $0.26 \pm 0.03$ & $4.0 \pm 0$ & $83 \pm 1.2$ & $133 \pm 27$ & $317 \pm 1$ \\
\hline 7 & $1: 7$ & 12 & 26 & 10.0 & $6.1 \pm 0$ & $0.26 \pm 0.03$ & $3.9 \pm 0$ & $88 \pm 0$ & $160 \pm 0$ & $290 \pm 0$ \\
\hline 8 & $1: 10$ & 6 & 15 & 8.0 & $6.8 \pm 0$ & $0.32 \pm 0.03$ & $4.3 \pm 0$ & $84 \pm 0$ & $133 \pm 27$ & $305 \pm 1$ \\
\hline 9 & $1: 7$ & 0 & 26 & 6.0 & $6.1 \pm 0$ & $0.46 \pm 0.07$ & $5.0 \pm 0$ & $111 \pm 0.7$ & $160 \pm 0$ & $296 \pm 1$ \\
\hline 10 & $1: 4$ & 18 & 37 & 4.0 & $3.3 \pm 0$ & $0.29 \pm 0$ & $3.9 \pm 0$ & $73 \pm 0.6$ & $107 \pm 27$ & $326 \pm 1$ \\
\hline 11 & $1: 10$ & 18 & 37 & 4.0 & $3.4 \pm 0$ & $0.23 \pm 0.12$ & $3.6 \pm 0$ & $77 \pm 0$ & $120 \pm 0$ & $344 \pm 1$ \\
\hline 12 & $1: 10$ & 18 & 15 & 8.0 & $6.8 \pm 0$ & $0.22 \pm 0.03$ & $3.5 \pm 0$ & $79 \pm 0$ & $133 \pm 27$ & $300 \pm 1$ \\
\hline 13 & $1: 4$ & 6 & 15 & 8.0 & $6.8 \pm 0$ & $0.36 \pm 0.03$ & $4.5 \pm 0$ & $87 \pm 0.4$ & $160 \pm 0$ & $306 \pm 0$ \\
\hline 14 & $1: 4$ & 6 & 15 & 4.0 & $3.9 \pm 0$ & $0.36 \pm 0.03$ & $4.5 \pm 0.1$ & $95 \pm 0.6$ & $120 \pm 40$ & $311 \pm 0$ \\
\hline 15 & $1: 10$ & 6 & 15 & 4.0 & $3.9 \pm 0$ & $0.26 \pm 0.03$ & $4.3 \pm 0$ & $82 \pm 0.1$ & $120 \pm 40$ & $306 \pm 0$ \\
\hline 16 & $1: 10$ & 18 & 15 & 4.0 & $3.9 \pm 0$ & $0.23 \pm 0.12$ & $3.5 \pm 0$ & $75 \pm 0.6$ & $120 \pm 40$ & $304 \pm 1$ \\
\hline 17 & $1: 7$ & 12 & 48 & 6.0 & $5.3 \pm 0$ & $0.29 \pm 0$ & $4.0 \pm 0$ & $84 \pm 0.1$ & $93 \pm 13$ & $362 \pm 1$ \\
\hline 18 & $1: 7$ & 12 & 26 & 2.0 & $3.0 \pm 0$ & $0.32 \pm 0.03$ & $4.0 \pm 0$ & $80 \pm 0.8$ & $107 \pm 27$ & $322 \pm 0$ \\
\hline 19 & $1: 10$ & 18 & 37 & 8.0 & $6.4 \pm 0$ & $0.22 \pm 0.03$ & $3.5 \pm 0$ & $79 \pm 0.2$ & $133 \pm 27$ & $310 \pm 2$ \\
\hline 20 & $1: 7$ & 12 & 26 & 6.0 & $5.6 \pm 0$ & $0.26 \pm 0.03$ & $3.8 \pm 0$ & $79 \pm 0.1$ & $133 \pm 40$ & $314 \pm 0$ \\
\hline 21 & $1: 7$ & 12 & 26 & 6.0 & $5.6 \pm 0$ & $0.23 \pm 0.09$ & $3.8 \pm 0$ & $85 \pm 0.1$ & $120 \pm 27$ & $313 \pm 0$ \\
\hline 22 & $1: 4$ & 6 & 37 & 8.0 & $6.5 \pm 0$ & $0.36 \pm 0.03$ & $4.5 \pm 0$ & $88 \pm 0.6$ & $133 \pm 40$ & $300 \pm 1$ \\
\hline 23 & $1: 13$ & 12 & 26 & 6.0 & $5.7 \pm 0$ & $0.09 \pm 0$ & $3.8 \pm 0$ & $67 \pm 0$ & $120 \pm 27$ & $298 \pm 0$ \\
\hline 24 & $1: 7$ & 12 & 26 & 6.0 & $5.6 \pm 0$ & $0.29 \pm 0.01$ & $4.0 \pm 0$ & $86 \pm 0$ & $107 \pm 27$ & $336 \pm 1$ \\
\hline 25 & $1: 4$ & 6 & 37 & 4.0 & $3.6 \pm 0$ & $0.36 \pm 0.03$ & $4.5 \pm 0$ & $92 \pm 0.6$ & $107 \pm 40$ & $329 \pm 0$ \\
\hline 26 & $1: 7$ & 12 & 26 & 6.0 & $5.6 \pm 0$ & $0.26 \pm 0.03$ & $3.7 \pm 0$ & $72 \pm 0$ & $120 \pm 27$ & $308 \pm 0$ \\
\hline 27 & $1: 4$ & 18 & 15 & 8.0 & $6.8 \pm 0$ & $0.23 \pm 0.12$ & $3.9 \pm 0$ & $75 \pm 0.1$ & $133 \pm 27$ & $299 \pm 1$ \\
\hline 28 & $1: 7$ & 12 & 26 & 6.0 & $5.6 \pm 0$ & $0.32 \pm 0.03$ & $3.8 \pm 0$ & $78 \pm 0.1$ & $107 \pm 27$ & $313 \pm 1$ \\
\hline 29 & $1: 4$ & 18 & 15 & 4.0 & $3.9 \pm 0$ & $0.23 \pm 0.12$ & $3.9 \pm 0$ & $77 \pm 0$ & $120 \pm 40$ & $298 \pm 0$ \\
\hline 30 & $1: 4$ & 18 & 37 & 8.0 & $6.5 \pm 0$ & $0.26 \pm 0.03$ & $3.8 \pm 0$ & $77 \pm 0.1$ & $120 \pm 40$ & $311 \pm 0$ \\
\hline
\end{tabular}

Note: TC, tannins content; PAC, phytic acid content; TIA, trypsin inhibitor activity; HA, hemagglutinating activity; TABC, total biogenic amines content.

*Values, showing mean \pm SE, were obtained from triplicate sets.

\section{Sensory Analysis}

Samples of idli, prepared under different experimental conditions of fermentation and optimized steaming time, were evaluated organoleptically by a panel of 10 trained judges. An overall sensory quality was considered using a 100-point score card (Table 5). Analysis of the samples was conducted in triplicate.

\section{Evaluation of Antinutrients}

While the samples of raw dal and rice were powdered, those of soaked dal and rice were made to paste using a blender (Bajaj Electricals, Mumbai, India). The powders, pastes and dal-rice mixed (unfermented and fermented) batters were left overnight at $-20 \mathrm{C}$, lyophilized (Eyela freeze dryer, FDU-506, Tokyo Rikakikai, Tokyo, Japan) and powdered.
The contents of tannins, phytic acid and total biogenic amines, and trypsin inhibitor and hemagglutinating activities were determined using the methods described by Price et al. (1978), Wheeler and Ferrel (1971), Yeh et al. (2006), Kakade et al. (1969) and Liener and Hill (1953), respectively. One unit $(\mathrm{U})$ of trypsin inhibitor activity was defined as a decrease in $\mathrm{A}_{280}$ of 0.01 , relative to the blank, in $20 \mathrm{~min}$ using a $10 \mathrm{~mL}$ assay volume, while one unit (U) of hemagglutinating activity was defined as the least amount of hemagglutinin which produced positive agglutination (1+) under the experimental condition. The analyses of the samples for estimating antinutrient levels were conducted in triplicate.

Following the traditional method of idli preparation, as described by Rakshit et al. (2015), the antinutrient levels of the product were evaluated to compare the efficiency of the optimized process conditions. 
TABLE 2. EXPERIMENTAL VALUES, BASED ON RSM DESIGN, OF ANTINUTRIENTS IN SOAKED RICE

\begin{tabular}{|c|c|c|c|c|c|c|c|c|}
\hline \multirow[b]{2}{*}{ Run } & \multicolumn{4}{|c|}{ Soaking condition (variable) } & \multirow[b]{2}{*}{ Final pH } & \multicolumn{3}{|c|}{ Antinutrient (per g dry wt)* } \\
\hline & $\begin{array}{l}\text { Rice:water } \\
(\mathrm{w} / \mathrm{w})\end{array}$ & $t(\mathrm{~h})$ & $T(\mathrm{C})$ & Initial pH & & $\mathrm{TC}(\mathrm{mg}) \dagger$ & PAC (mg) & TBAC $(\mu \mathrm{g})$ \\
\hline 1 & $1: 1$ & 12 & 26 & 6.0 & $5.8 \pm 0$ & $0.06 \pm 0.03$ & $1.3 \pm 0$ & $81 \pm 2.2$ \\
\hline 2 & $1: 7$ & 12 & 4 & 6.0 & $5.9 \pm 0$ & $0.03 \pm 0.03$ & $1.1 \pm 0$ & $76 \pm 1.7$ \\
\hline 3 & $1: 7$ & 24 & 26 & 6.0 & $4.9 \pm 0$ & $<\mathrm{dl}$ & $0.9 \pm 0$ & $78 \pm 2.9$ \\
\hline 4 & $1: 10$ & 6 & 37 & 4.0 & $3.8 \pm 0$ & $<\mathrm{dl}$ & $1.3 \pm 0$ & $98 \pm 0$ \\
\hline 5 & $1: 10$ & 6 & 37 & 8.0 & $6.4 \pm 0$ & $0.03 \pm 0.03$ & $1.3 \pm 0$ & $79 \pm 0.9$ \\
\hline 6 & $1: 7$ & 12 & 26 & 6.0 & $5.8 \pm 0$ & $<\mathrm{dl}$ & $1.4 \pm 0$ & $77 \pm 2.3$ \\
\hline 7 & $1: 7$ & 12 & 26 & 10.0 & $6.2 \pm 0$ & $0.03 \pm 0.03$ & $1.3 \pm 0$ & $79 \pm 1.0$ \\
\hline 8 & $1: 10$ & 6 & 15 & 8.0 & $6.6 \pm 0$ & $<\mathrm{dl}$ & $1.3 \pm 0$ & $75 \pm 0$ \\
\hline 9 & $1: 7$ & 0 & 26 & 6.0 & $6.1 \pm 0$ & $0.06 \pm 0.03$ & $1.4 \pm 0$ & $76 \pm 1.0$ \\
\hline 10 & $1: 4$ & 18 & 37 & 4.0 & $3.3 \pm 0$ & $<\mathrm{dl}$ & $1.4 \pm 0$ & $96 \pm 1.2$ \\
\hline 11 & $1: 10$ & 18 & 37 & 4.0 & $3.2 \pm 0$ & $<\mathrm{dl}$ & $1.1 \pm 0$ & $86 \pm 0.9$ \\
\hline 12 & $1: 10$ & 18 & 15 & 8.0 & $6.6 \pm 0$ & $<\mathrm{dl}$ & $1.2 \pm 0$ & $73 \pm 1.5$ \\
\hline 13 & $1: 4$ & 6 & 15 & 8.0 & $6.7 \pm 0$ & $0.06 \pm 0.03$ & $1.5 \pm 0$ & $80 \pm 0$ \\
\hline 14 & $1: 4$ & 6 & 15 & 4.0 & $3.8 \pm 0$ & $0.03 \pm 0$ & $1.6 \pm 0$ & $87 \pm 0.8$ \\
\hline 15 & $1: 10$ & 6 & 15 & 4.0 & $3.8 \pm 0$ & $0.03 \pm 0.03$ & $1.3 \pm 0$ & $82 \pm 0.7$ \\
\hline 16 & $1: 10$ & 18 & 15 & 4.0 & $3.7 \pm 0.1$ & $<\mathrm{dl}$ & $0.7 \pm 0$ & $78 \pm 1.0$ \\
\hline 17 & $1: 7$ & 12 & 48 & 6.0 & $4.4 \pm 0$ & $0.03 \pm 0.03$ & $1.0 \pm 0$ & $95 \pm 1.1$ \\
\hline 18 & $1: 7$ & 12 & 26 & 2.0 & $3.0 \pm 0$ & $<\mathrm{dl}$ & $1.2 \pm 0$ & $97 \pm 1.7$ \\
\hline 19 & $1: 10$ & 18 & 37 & 8.0 & $5.9 \pm 0$ & $<\mathrm{dl}$ & $1.0 \pm 0$ & $81 \pm 0.9$ \\
\hline 20 & $1: 7$ & 12 & 26 & 6.0 & $5.9 \pm 0$ & $0.06 \pm 0.03$ & $1.1 \pm 0$ & $73 \pm 6.0$ \\
\hline 21 & $1: 7$ & 12 & 26 & 6.0 & $5.9 \pm 0$ & $0.03 \pm 0$ & $1.3 \pm 0$ & $74 \pm 0$ \\
\hline 22 & $1: 4$ & 6 & 37 & 8.0 & $6.6 \pm 0$ & $0.06 \pm 0.03$ & $1.4 \pm 0$ & $84 \pm 0.2$ \\
\hline 23 & $1: 13$ & 12 & 26 & 6.0 & $5.9 \pm 0$ & $<\mathrm{dl}$ & $0.9 \pm 0$ & $80 \pm 0.1$ \\
\hline 24 & $1: 7$ & 12 & 26 & 6.0 & $5.9 \pm 0$ & $<\mathrm{dl}$ & $0.9 \pm 0$ & $80 \pm 0$ \\
\hline 25 & $1: 4$ & 6 & 37 & 4.0 & $3.7 \pm 0$ & $0.03 \pm 0.03$ & $1.4 \pm 0.1$ & $96 \pm 0.2$ \\
\hline 26 & $1: 7$ & 12 & 26 & 6.0 & $5.9 \pm 0$ & $0.06 \pm 0.03$ & $1.0 \pm 0$ & $73 \pm 0.9$ \\
\hline 27 & $1: 4$ & 18 & 15 & 8.0 & $6.6 \pm 0$ & $<\mathrm{dl}$ & $1.1 \pm 0$ & $82 \pm 0.3$ \\
\hline 28 & $1: 7$ & 12 & 26 & 6.0 & $5.9 \pm 0$ & $<\mathrm{dl}$ & $1.1 \pm 0$ & $79 \pm 0.1$ \\
\hline 29 & $1: 4$ & 18 & 15 & 4.0 & $3.8 \pm 0$ & $<\mathrm{dl}$ & $1.5 \pm 0$ & $79 \pm 0.1$ \\
\hline 30 & $1: 4$ & 18 & 37 & 8.0 & $6.0 \pm 0$ & $<\mathrm{dl}$ & $1.4 \pm 0$ & $81 \pm 0.4$ \\
\hline
\end{tabular}

Note: TC, tannins content; PAC, phytic acid content; TABC, total biogenic amines content.

*Values, showing mean $\pm \mathrm{SE}$, were obtained from triplicate sets.

${ }^{\dagger} \mathrm{dl}$ (detection limit), $0.003 \mathrm{mg} / \mathrm{g}$ dry wt.

\section{Validation of the Model Equations}

Following numerical optimization, the optimized models of each processing stage were subjected to validation. The deviation error was expressed as a percentage, which was computed by comparing the actual values with the predicted ones at the optimum condition to evaluate model performance.

\section{RESULTS AND DISCUSSION}

\section{Raw Dal/Rice}

While raw dal contained considerable amounts of antinutrients, rice had a lesser amount of those (Table 6). The values of tannins content, phytic acid content and hemagglutinating activity of raw dal and rice were similar to earlier reports (Hettiarachchy and Sri Kantha 1981; Hemalatha et al. 2007).

\section{Soaking}

Results for levels of antinutrients under different soaking conditions of dal according to RSM design are shown in Table 1. The models have significant $F$ value, an insignificant lack-of-fit, low-standard deviation and coefficient of variance (Table 7). The low coefficients of determination $\left(R^{2}\right)$ values for hemagglutinating and trypsin inhibitor activities show that 54 and $59 \%$ of variations are influenced by independent variables of soaking conditions, and the remaining variations can be attributed to other factors. The adjusted and predicted $R^{2}$ for models are in reasonable agreement. Adequate precision for models in the study has ratio greater than 4 , which is desirable and indicates adequate model discrimination. The low values of $C V, S D$ and PRESS for the models show adequacy with which the experiment is conducted and indicate a better prediction. 


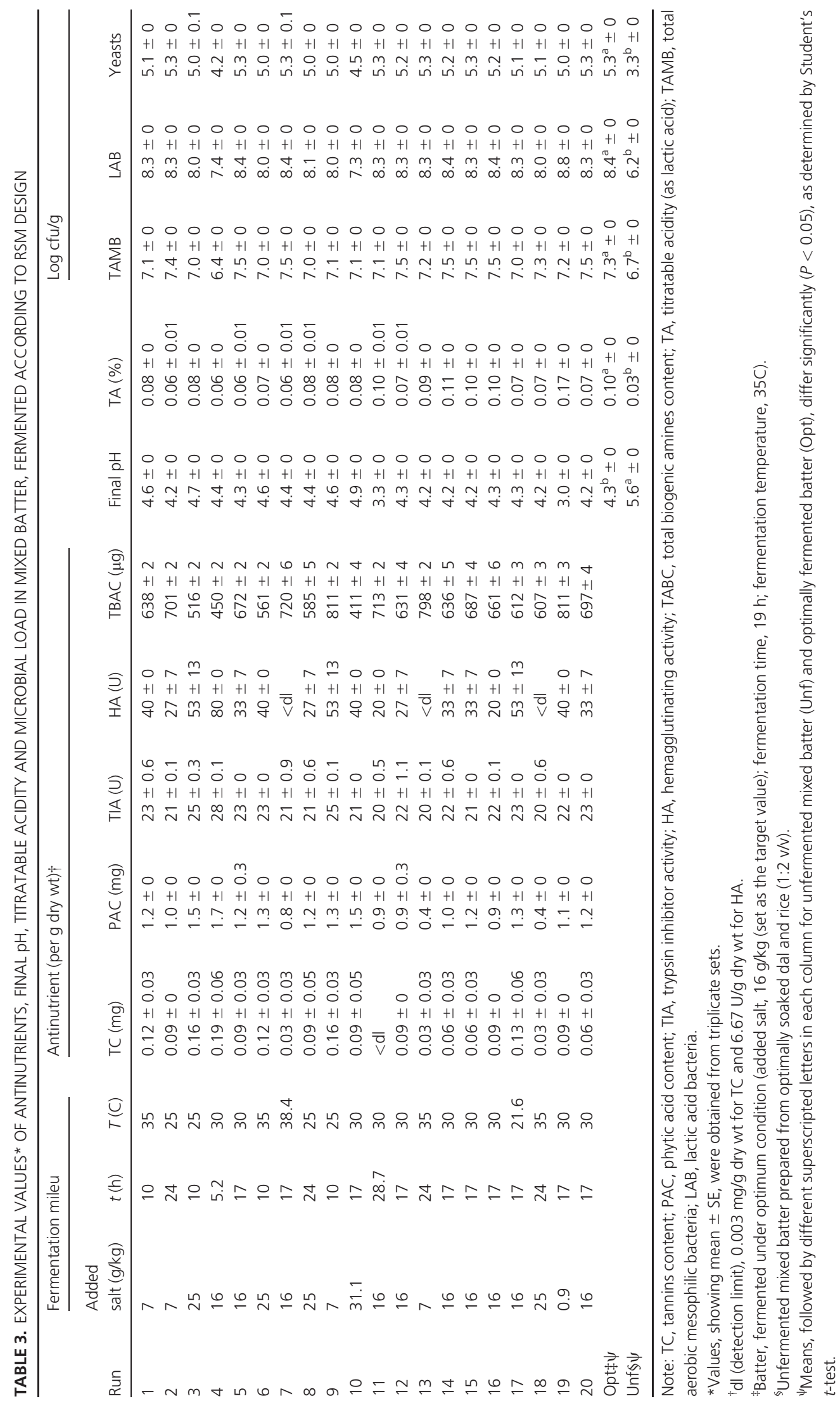


TABLE 4. EXPERIMENTAL VALUES OF ANTINUTRIENTS IN FERMENTED BATTER, STEAMED ACCORDING TO RSM DESIGN

\begin{tabular}{|c|c|c|c|c|c|c|}
\hline \multirow[b]{2}{*}{ Run } & \multirow[b]{2}{*}{$\begin{array}{l}\text { Steaming } \\
\text { time (min) }\end{array}$} & \multicolumn{5}{|c|}{ Antinutrient (per g dry $w t)^{*}$} \\
\hline & & $\begin{array}{l}\text { TC } \\
(\mathrm{mg}) \dagger\end{array}$ & PAC (mg) & $\mathrm{TIA}(\mathrm{U})$ & $\begin{array}{l}\mathrm{HA} \\
(\mathrm{U}) \dagger\end{array}$ & $\begin{array}{l}\text { TBAC } \\
(\mu \mathrm{g})\end{array}$ \\
\hline 1 & 20 & $<\mathrm{dl}$ & $0.3 \pm 0.02$ & $17 \pm 0.1$ & $<\mathrm{dl}$ & $636 \pm 2$ \\
\hline 2 & 15 & $<\mathrm{dl}$ & $0.4 \pm 0$ & $18 \pm 0$ & $<\mathrm{dl}$ & $639 \pm 1$ \\
\hline 3 & 12.5 & $<\mathrm{dl}$ & $0.6 \pm 0$ & $18 \pm 0$ & $<\mathrm{dl}$ & $644 \pm 5$ \\
\hline 4 & 20 & $<\mathrm{dl}$ & $0.3 \pm 0.02$ & $17 \pm 0.1$ & $<\mathrm{dl}$ & $636 \pm 0$ \\
\hline 5 & 17.5 & $<\mathrm{dl}$ & $0.3 \pm 0.02$ & $18 \pm 0$ & $<\mathrm{dl}$ & $635 \pm 3$ \\
\hline 6 & 10 & $<\mathrm{dl}$ & $0.4 \pm 0$ & $19 \pm 0.1$ & $<\mathrm{dl}$ & $643 \pm 1$ \\
\hline 7 & 10 & $<\mathrm{dl}$ & $0.4 \pm 0$ & $19 \pm 0.1$ & $<\mathrm{dl}$ & $643 \pm 4$ \\
\hline
\end{tabular}

Note: TC, tannins content; PAC, phytic acid content; TIA, trypsin inhibitor activity; $\mathrm{HA}$, hemagglutinating activity; $\mathrm{TABC}$, total biogenic amines content.

*Values, showing mean $\pm \mathrm{SE}$, were obtained from triplicate sets.

${ }^{\dagger} \mathrm{dl}$ (detection limit), $0.003 \mathrm{mg} / \mathrm{g}$ dry wt for TC and $6.67 \mathrm{U} / \mathrm{g}$ dry wt for HA.

The reduction $(P<0.05)$ of tannins content, phytic acid content and trypsin inhibitor activity was influenced by dalwater ratio and time. Although the reduction of hemagglutinating activity was dependent on temperature, initial $\mathrm{pH}$ had no effect $(P<0.05)$. While initial $\mathrm{pH}$ played a significant $(P<0.05)$ role in the reduction of total biogenic amines content, temperature had an opposite $(P<0.05)$ effect. Interestingly, interaction of initial $\mathrm{pH}$ and temperature reduced $(P<0.05)$ the total biogenic amines content. After removing insignificant terms for the coded variables of each response, the reduced polynomial equations were:

Tannins content $(\mathrm{mg} / \mathrm{g})=0.283-0.044 A-0.054 B$

Phytic acid content $(\mathrm{mg} / \mathrm{g})=3.844-0.189 A-$ $0.378 B+0.119 A^{2}$

Trypsin inhibitor activity $(\mathrm{U} / \mathrm{g})=82.598-4.380 \mathrm{~A}-$ $7.327 B$

Hemagglutinating activity $(\mathrm{U} / \mathrm{g})=125.756-6.611 C+$ $10.972 D$

Total biogenic amines content $(\mu \mathrm{g} / \mathrm{g})=316.816+$ $9.709 C-7.042 D-5.547 C D-4.724 A^{2}-3.615 B^{2}+$ $4.454 C^{2}$

where $A$ was dal-water ratio, $B$ was time, $C$ was temperature and $D$ was initial $\mathrm{pH}$.

An increase in time and dal-water ratio caused a linear reduction in tannins content $(0.19 \mathrm{mg} / \mathrm{g}$; Fig. 1a), phytic acid content (3.4 mg/g; Fig. 1c), trypsin inhibitor activity (71 U/g; Fig. 1e) and hemagglutinating activity $(118 \mathrm{U} / \mathrm{g}$; Fig. 1f) when temperature and initial $\mathrm{pH}$ were kept constant at $26 \mathrm{C}$ and 6.0, respectively. The predicted reduction of tannins content, phytic acid content, trypsin inhibitor activity

TABLE 5. SENSORY SCORES OF IDLI, OBTAINED THROUGH DIFFERENT FERMENTATION CONDITIONS

\begin{tabular}{|c|c|c|c|c|c|c|c|c|c|}
\hline \multirow[b]{2}{*}{ Run } & \multicolumn{3}{|c|}{ Fermentation condition } & \multicolumn{6}{|c|}{ Sensory attribute } \\
\hline & $\begin{array}{l}\text { Added } \\
\text { salt ( } / \mathrm{kg})\end{array}$ & $\begin{array}{l}\text { Time } \\
\text { (h) }\end{array}$ & $\begin{array}{l}\text { Temperature } \\
\text { (C) }\end{array}$ & $\begin{array}{l}\text { Taste (max. } \\
\text { score, } 35 \text { ) }\end{array}$ & $\begin{array}{l}\text { Flavor (max. } \\
\text { score, } 30 \text { ) }\end{array}$ & $\begin{array}{l}\text { Body and } \\
\text { texture (max. } \\
\text { score, } 30)\end{array}$ & $\begin{array}{l}\text { Color (max. } \\
\text { score, } 5 \text { ) }\end{array}$ & $\begin{array}{l}\text { Overall quality } \\
\text { (max. score, 100) }\end{array}$ & Grade* \\
\hline 1 & 7 & 10 & 35 & $23 \pm 1$ & $24 \pm 1$ & $28 \pm 0$ & $4 \pm 0$ & $79 \pm 2$ & $\mathrm{~F}$ \\
\hline 2 & 7 & 24 & 25 & $22 \pm 1$ & $29 \pm 0$ & $28 \pm 0$ & $3 \pm 0$ & $82 \pm 1$ & G \\
\hline 3 & 25 & 10 & 25 & $29 \pm 0$ & $25 \pm 0$ & $28 \pm 0$ & $3 \pm 0$ & $85 \pm 0$ & G \\
\hline 4 & 16 & 5.2 & 30 & $27 \pm 0$ & $17 \pm 1$ & $28 \pm 0$ & $4 \pm 0$ & $76 \pm 1$ & $F$ \\
\hline 5 & 16 & 17 & 30 & $32 \pm 0$ & $28 \pm 1$ & $28 \pm 0$ & $3 \pm 0$ & $91 \pm 1$ & $E$ \\
\hline 6 & 25 & 10 & 35 & $28 \pm 0$ & $24 \pm 1$ & $27 \pm 0$ & $4 \pm 0$ & $83 \pm 1$ & G \\
\hline 7 & 16 & 17 & 38.4 & $31 \pm 1$ & $26 \pm 0$ & $28 \pm 0$ & $4 \pm 0$ & $89 \pm 1$ & G \\
\hline 8 & 25 & 24 & 25 & $27 \pm 0$ & $28 \pm 0$ & $28 \pm 0$ & $4 \pm 0$ & $87 \pm 0$ & G \\
\hline 9 & 7 & 10 & 25 & $22 \pm 0$ & $24 \pm 0$ & $28 \pm 0$ & $4 \pm 0$ & $78 \pm 0$ & $F$ \\
\hline 10 & 31.1 & 17 & 30 & $26 \pm 1$ & $28 \pm 0$ & $28 \pm 1$ & $3 \pm 0$ & $85 \pm 2$ & G \\
\hline 11 & 16 & 28.7 & 30 & $33 \pm 0$ & $28 \pm 0$ & $28 \pm 0$ & $4 \pm 0$ & $93 \pm 0$ & $E$ \\
\hline 12 & 16 & 17 & 30 & $33 \pm 0$ & $28 \pm 0$ & $28 \pm 0$ & $3 \pm 0$ & $92 \pm 0$ & $E$ \\
\hline 13 & 7 & 24 & 35 & $31 \pm 0$ & $29 \pm 0$ & $28 \pm 0$ & $4 \pm 0$ & $92 \pm 0$ & G \\
\hline 14 & 16 & 17 & 30 & $32 \pm 0$ & $28 \pm 0$ & $28 \pm 0$ & $4 \pm 0$ & $92 \pm 0$ & G \\
\hline 15 & 16 & 17 & 30 & $31 \pm 0$ & $28 \pm 0$ & $29 \pm 0$ & $4 \pm 0$ & $92 \pm 0$ & $E$ \\
\hline 16 & 16 & 17 & 30 & $32 \pm 1$ & $29 \pm 0$ & $28 \pm 0$ & $3 \pm 0$ & $92 \pm 1$ & $E$ \\
\hline 17 & 16 & 17 & 21.6 & $32 \pm 0$ & $29 \pm 0$ & $29 \pm 0$ & $3 \pm 0$ & $93 \pm 0$ & $E$ \\
\hline 18 & 25 & 24 & 35 & $29 \pm 0$ & $29 \pm 0$ & $29 \pm 0$ & $4 \pm 0$ & $91 \pm 0$ & G \\
\hline 19 & 0.9 & 17 & 30 & $24 \pm 1$ & $28 \pm 0$ & $27 \pm 0$ & $3 \pm 0$ & $82 \pm 1$ & G \\
\hline 20 & 16 & 17 & 30 & $31 \pm 1$ & $28 \pm 0$ & $29 \pm 0$ & $3 \pm 0$ & $91 \pm 1$ & $E$ \\
\hline Opt. $\dagger$ & 16 & 19 & 35 & $32 \pm 0$ & $29 \pm 0$ & $29 \pm 0$ & $4 \pm 0$ & $94 \pm 0$ & $E$ \\
\hline
\end{tabular}

Note: Values are mean $\pm \mathrm{SE}(n=30)$.

${ }^{*} F$, fair; $G$, good; $E$, excellent.

${ }^{\dagger}$ Optimum condition, set by targeting $16 \mathrm{~g} / \mathrm{kg}$ and $19 \mathrm{~h}$ as the desired salt level and fermentation time, respectively. 
TABLE 6. ANTINUTRIENTS IN SUBSTRATES AT OPTIMUM CONDITION OF EACH STAGE OF IDLI PREPARATION

\begin{tabular}{|c|c|c|c|c|c|}
\hline \multirow[b]{2}{*}{ Parameter } & \multicolumn{5}{|c|}{ Antinutrient (per g dry wt) } \\
\hline & $\mathrm{TC}(\mathrm{mg}) \ddagger$ & PAC (mg) & $\mathrm{TIA}(\mathrm{U}) \ddagger$ & $\mathrm{HA}(\mathrm{U}) \neq$ & TBAC $(\mu \mathrm{g})$ \\
\hline Raw dal* & $0.49^{\mathrm{A}} \pm 0.06$ & $5.1^{\mathrm{A}} \pm 0$ & $120^{\mathrm{A}} \pm 18.5$ & $160^{A} \pm 0$ & $295^{B} \pm 3$ \\
\hline \multicolumn{6}{|c|}{ Soaking (Raw Dal-Water of 1:5 W/W, pH 4.0, 16C, 18 h) } \\
\hline Predicted values & 0.25 & 3.8 & 78 & 119 & 301 \\
\hline Experimental values* & $0.26^{B} \pm 0.03$ & $3.8^{\mathrm{A}} \pm 0$ & $81^{B} \pm 8.6$ & $120^{B} \pm 0$ & $303^{A} \pm 3$ \\
\hline Error \% & 3.85 & 0 & 3.7 & 0.83 & 0.66 \\
\hline$\%$ change $\dagger$ & -49.0 & -25.5 & -35.0 & -25.6 & +2.0 \\
\hline Raw rice* & $0.06^{x} \pm 0.03$ & $1.5^{x} \pm 0$ & $<\mathrm{dl}$ & $<\mathrm{dl}$ & $70^{Y} \pm 4$ \\
\hline \multicolumn{6}{|c|}{ Soaking (Raw Rice-Water of 1:5 W/W, pH 5.6, 16C, 18 h) } \\
\hline Predicted values & 0.01 & 1.2 & 0 & 0 & 75 \\
\hline Experimental values* & $<\mathrm{dl}^{\mathrm{Y}}$ & $1.1^{x} \pm 0$ & $<\mathrm{dl}$ & $<\mathrm{dl}$ & $76^{x} \pm 2$ \\
\hline Error \% & 0 & 9.09 & 0 & 0 & 1.32 \\
\hline$\%$ change $\dagger$ & -83.3 & -20.0 & 0 & 0 & +7.1 \\
\hline \multicolumn{6}{|c|}{ Unfermented Mixed Batter (Dal Batter: Rice Slurry; 1:2 v/v) } \\
\hline Experimental values* & $0.09^{1} \pm 0$ & $1.7^{1} \pm 0.1$ & $33^{1} \pm 4.8$ & $40^{1} \pm 0$ & $127^{3} \pm 3$ \\
\hline \multicolumn{6}{|c|}{ Fermentation (Salt Concentration of $16 \mathrm{~g} / \mathrm{kg}, 35 \mathrm{C}, 19 \mathrm{~h}$ ) } \\
\hline Predicted values & 0.05 & 0.8 & 21 & 14 & 681 \\
\hline Experimental values* & $0.05^{2} \pm 0$ & $0.7^{2} \pm 0$ & $21^{2} \pm 2.7$ & $14^{2} \pm 0$ & $681^{1} \pm 1$ \\
\hline Error \% & 0 & 14.3 & 0 & 0 & 0 \\
\hline \% change† & -44.4 & -53 & -36.4 & -65 & +436.2 \\
\hline \multicolumn{6}{|l|}{ Steaming (20 min) } \\
\hline Predicted values & 0 & 0.3 & 17 & 0 & 636 \\
\hline Experimental values* & $<\mathrm{dl}$ & $0.3^{3} \pm 0$ & $17^{2} \pm 0.4$ & $<\mathrm{dl}$ & $636^{2} \pm 0$ \\
\hline Error \% & 0 & 0 & 0 & 0 & 0 \\
\hline$\%$ change $\dagger$ & $-100(-100)$ & $-82.4(-62.5)$ & $-48.5(-19)$ & $-100(-100)$ & $+400.8(-6.6)$ \\
\hline
\end{tabular}

Note: TC, tannins content; PAC, phytic acid content; TIA, trypsin inhibitor activity; HA, hemagglutinating activity; TABC, total biogenic amines content.

*Values are mean $\pm S E$ of triplicate determinations in raw/processed samples. Means followed by the different superscripts in each column for raw and soaked dal $(A$ and $B) /$ rice $(X$ and $Y)$ differ significantly $(P<0.05)$ as determined by $t$-test, and for unfermented, fermented and steamed dal-rice mixture (1, 2 and 3$)$ differ significantly $(P<0.05)$ as determined by Duncan multiple range test.

${ }^{\dagger}-$ and + indicate percent decrease and increase, respectively, of predicted values over raw dal/rice for the soaking stage, and over unfermented mixed batter for fermentation and steaming stages (values within parentheses indicate percentages calculated over the predicted values of fermented batter).

"dl (detection limit), $0.003 \mathrm{mg} / \mathrm{g}$ dry wt for TC, $15.26 \mathrm{U} / \mathrm{g}$ dry wt for TIA and $6.67 \mathrm{U} / \mathrm{g}$ dry wt for HA.

and hemagglutinating activity over raw dal was $61,33,41$ and $26 \%$, respectively. The minimum total biogenic amines content (308 $\mu \mathrm{g} / \mathrm{g}$; Fig. 1g) was obtained when dal was soaked in water of initial $\mathrm{pH} 8.0$ and $20.5 \mathrm{C}$, keeping dalwater ratio and time constant at 1:7 and $12 \mathrm{~h}$, respectively.

The loss of tannins content is attributed to the binding of tannins with carbohydrates and proteins, and also to the activation of enzyme polyphenol oxidase (Saharan et al. 2002). Therefore, an increase in dal-water ratio and time might have enhanced leaching of tannins and phytate from dal (beans having no external barrier, i.e., seed coat) into the soak water. The loss of trypsin inhibitor activity might be due to leaching of trypsin inhibitors. Since trypsin inhibitors are low-molecular weight proteins, they are likely to leach out of the seeds easily (Grewal and Jood 2006). The reduction of tannins content, phytic acid content, trypsin inhibitor activity and hemagglutinating activity during soaking in the present study is in agreement with the earlier reports (Grewal and Jood 2006; Khandelwal et al. 2010; Kalpanadevi and Mohan 2013). The level of total biogenic amines content reduced with an increase in initial $\mathrm{pH}$ and a decrease in temperature. For the production of decarboxylase, a low pH (3.0-6.0) is favorable to bacteria (Silla Santos 1996). A higher temperature favors proteolysis and decarboxylation of amino acids, resulting in an increased concentration of amines (Joosten and van Boekel 1988).

Results for levels of antinutrients under different soaking conditions of raw rice according to RSM design are shown in Table 2. In case of rice soaking, the models for different antinutrients are significant with nonsignificant lack of fit (Table 7). For the contents of tannins and phytic acid, model terms explain $50 \%$ of variations. On the other hand, $R^{2}$ value of total biogenic amines indicates a good fit between predicted values and the experimental data points. This implies that $92 \%$ of the variations for reduction in the antinutrient levels are 
TABLE 7. GOODNESS OF FIT OF THE MODELS GENERATED

\begin{tabular}{|c|c|c|c|c|c|c|c|c|}
\hline Model & $R^{2}$ & $\operatorname{Adj} R^{2}$ & Pred $R^{2}$ & Adeq precision & $S D$ & Mean & $C V \%$ & PRESS \\
\hline \multicolumn{9}{|c|}{ Soaking of Blackgram } \\
\hline $\mathrm{TC}$ & 0.724 & 0.680 & 0.587 & 13.033 & 0.043 & 0.283 & 15.113 & 0.068 \\
\hline PAC & 0.934 & 0.872 & 0.683 & 14.289 & 0.149 & 4.010 & 3.732 & 1.605 \\
\hline TIA & 0.593 & 0.528 & 0.391 & 10.344 & 6.940 & 82.590 & 8.402 & 1803.151 \\
\hline $\mathrm{HA}$ & 0.539 & 0.466 & 0.331 & 9.839 & 12.641 & 125.756 & 10.052 & 5803.069 \\
\hline TBAC & 0.873 & 0.754 & 0.564 & 11.069 & 7.727 & 311.766 & 2.479 & 3063.573 \\
\hline \multicolumn{9}{|c|}{ Soaking of Rice } \\
\hline $\mathrm{TC}$ & 0.500 & 0.42 & 0.351 & 8.018 & 0.018 & 0.020 & 91.652 & 0.011 \\
\hline PAC & 0.501 & 0.421 & 0.301 & 7.279 & 0.163 & 1.217 & 13.419 & 0.934 \\
\hline TBAC & 0.915 & 0.836 & 0.636 & 10.642 & 3.064 & 81.799 & 3.746 & 603.469 \\
\hline \multicolumn{9}{|c|}{ Fermentation } \\
\hline $\mathrm{TC}$ & 0.861 & 0.835 & 0.805 & 17.745 & 0.019 & 0.091 & 21.658 & 0.009 \\
\hline PAC & 0.747 & 0.699 & 0.569 & 13.001 & 0.183 & 1.085 & 16.856 & 0.912 \\
\hline TIA & 0.953 & 0.911 & 0.769 & 17.947 & 0.626 & 22.469 & 2.784 & 19.268 \\
\hline $\mathrm{HA}$ & 0.892 & 0.871 & 0.817 & 19.751 & 7.469 & 31.667 & 23.567 & 1510.470 \\
\hline TBAC & 0.793 & 0.754 & 0.653 & 15.425 & 50.357 & 633.707 & 7.947 & 67966.570 \\
\hline \multicolumn{9}{|l|}{ Steaming } \\
\hline PAC & 1 & 1 & NA & - & 0 & 1.187 & 0 & NA \\
\hline $\mathrm{TIA}$ & 0.981 & 0.977 & 0.963 & 28.253 & 0.329 & 22.416 & 1.470 & 1.054 \\
\hline TBAC & 0.998 & 0.996 & 0.983 & 52.986 & 0.234 & 639.366 & 0.037 & 1.571 \\
\hline
\end{tabular}

Note: TC, tannins content; PAC, phytic acid content; TIA, trypsin inhibitor activity; HA, hemagglutinating activity; TABC, total biogenic amines content; $S D$, standard deviation; CV, coefficient of variation; PRESS, predicated residual error sum of squares; NA, not applicable.

explained by independent variables studied in the present study. Regression coefficient data for the soaking of rice indicate rice-water ratio and time caused a reduction $(P<0.05)$ of the contents of tannins and phytic acid. The main influencing factors for reduction of total biogenic amines content were rice-water ratio, initial $\mathrm{pH}$ and interaction of temperature and initial $\mathrm{pH}$. However, temperature alone showed an opposite $(P<0.05)$ effect. The reduced polynomial equations were:

Tannins content $(\mathrm{mg} / \mathrm{g})=0.020-0.010 A-0.015 B$

Phytic acid content $(\mathrm{mg} / \mathrm{g})=1.217-0.119 A-0.116 B$

Total biogenic amines content $(\mu \mathrm{g} / \mathrm{g})=75.946-1.467 \mathrm{~A}$ $+4.331 C-4.390 D-2.242 C D+2.486 C^{2}+3.191 D^{2}$ where $A$ was rice-water ratio, $B$ was time, $C$ was temperature and $D$ was initial $\mathrm{pH}$.

Keeping temperature and $\mathrm{pH}$ constant at 26C and 6.0, respectively, an increase in time and rice-water ratio caused a complete reduction in tannins content (Fig. 1b); the level of phytic acid content reduced to $0.98 \mathrm{mg} / \mathrm{g}$ (Fig. 1d), indicating 35\% reduction over raw rice. The minimum content of total biogenic amines ( $74 \mu \mathrm{g} / \mathrm{g}$; Fig. 1h) was obtained by soaking rice at $20.5 \mathrm{C}$ in water $(\mathrm{pH} 7.0)$, and keeping the rice-water ratio fixed at 1:7 and time at $12 \mathrm{~h}$.

\section{Optimized Soaking Condition}

After numerical optimization, the optimum condition for soaking of dal was 1:10 of dal-water ratio, time of $18 \mathrm{~h}$, temperature of $16 \mathrm{C}$ and initial $\mathrm{pH}$ of 4.0 , and that for soaking of rice was 1:10 of rice-water ratio, time of $18 \mathrm{~h}$, temperature of $22 \mathrm{C}$ and initial $\mathrm{pH}$ of 7.1. To minimize wastage of water, desirability of various target ratios was compared $(P<0.01)$ and a ratio of 1:5 for dal/rice-water was selected, Therefore, the optimized soaking condition for dal was 1:5 of dal-water, $18 \mathrm{~h}, 16 \mathrm{C}$ and initial $\mathrm{pH} 4.0$. Similarly, the optimum soaking condition for rice was 1:5 of rice-water, $18 \mathrm{~h}, 16 \mathrm{C}$ and initial $\mathrm{pH}$ 5.6. Predicted reduction $(P<$ 0.05 ) of antinutrient levels for soaked dal and rice is shown in Table 6.

The initial $\mathrm{pH}$ (set under different experimental conditions) for both dal and rice ranged from 4 to 10 . The final $\mathrm{pH}$ of soak water of dal and rice ranged from 3.0 to 6.8 and 3.0 to 6.6 (run numbers: 18 to 8 ), respectively (Tables 1 and 2 ). In the present study, the decrease in $\mathrm{pH}$ might be due to accumulation of organic acids by the growth of microbiota during soaking (Ashenafi and Busse 1991).

\section{Unfermented Mixed Batter}

Optimally soaked dal paste and rice slurry were mixed in a ratio of 1:2 v/v to prepare unfermented mixed batter. Compared to dal, rice contained negligible amounts of antinutrients. Since in the mixed batter dal constituted one-third by volume, the level of antinutrients in mixed batter was approximately one-third of the level of those in soaked dal (Table 6). 
FIG. 1. RESPONSE SURFACE 3D PLOTS SHOWING THE INFLUENCE OF BLACKGRAM DAL/RICE-WATER RATIO, SOAKING TIME $(t)$, SOAKING TEMPERATURE (T) AND INITIAL PH OF SOAKING WATER ON ANTINUTRIENTS: INFLUENCE OF DAL/RICE-WATER RATIO AND $t$ ON TANNINS CONTENT (TC; a and b), PHYTIC ACID CONTENT (PAC; $c$ and d), TRYPSIN INHIBITOR ACTIVITY (TIA; e) AND HEMAGGLUTINATING ACTIVITY (HA; f) WHEN TAND PH WERE KEPT CONSTANT AT 26C AND 6.0, RESPECTIVELY; INFLUENCE OF T AND $\mathrm{pH}$ ON TOTAL BIOGENIC AMINES CONTENT (TBAC; $g$ and $h$ ) WHEN DAL/RICE-WATER RATIO AND $t$ WERE KEPT CONSTANT AT 1:7 AND $12 \mathrm{~h}$, RESPECTIVELY a
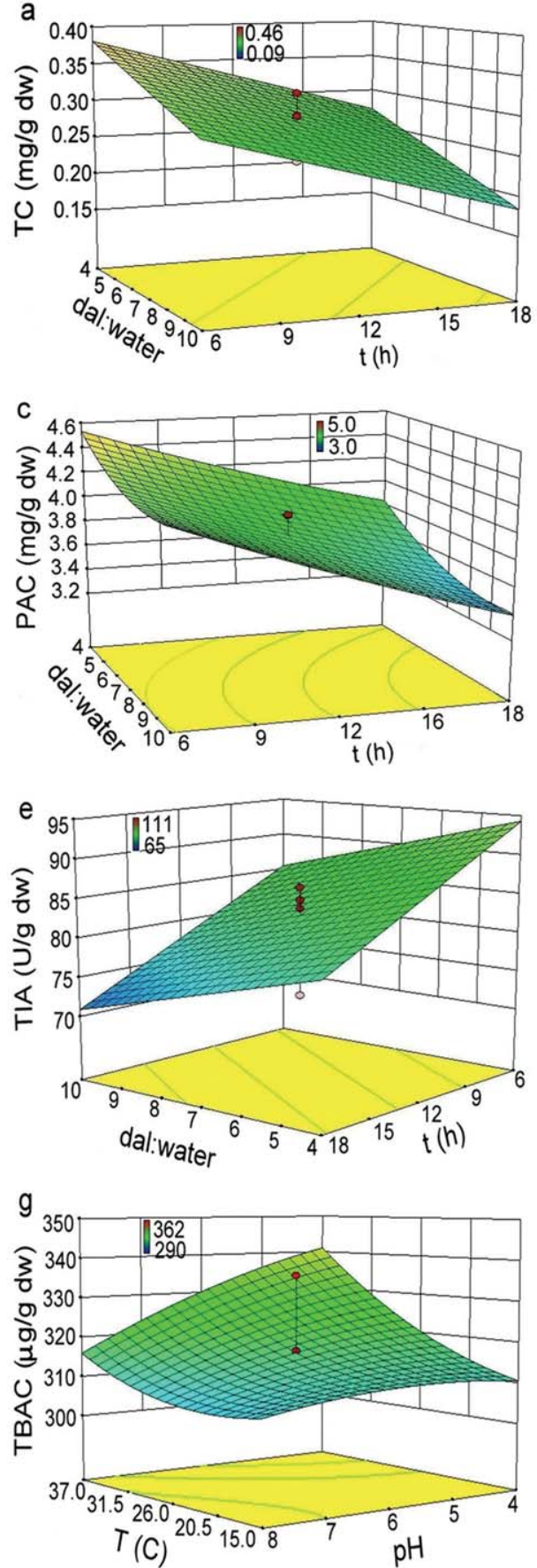
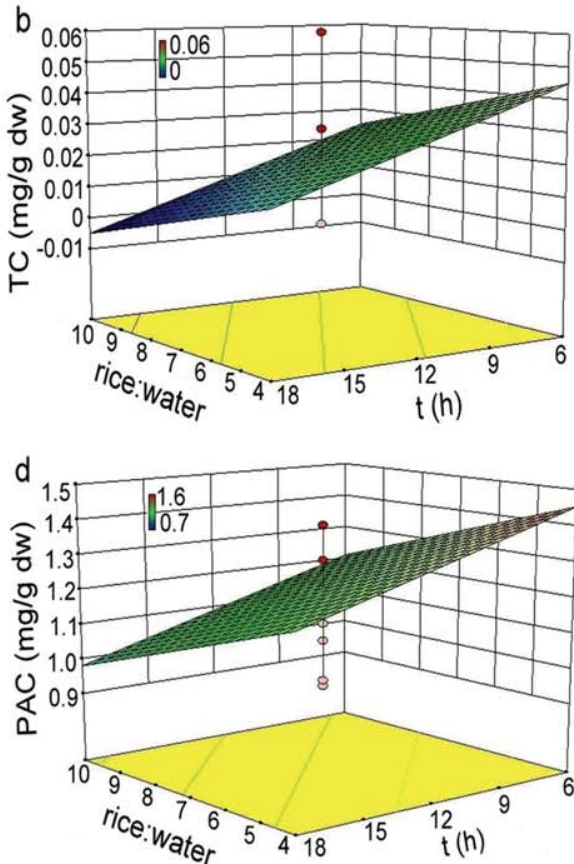

$f$
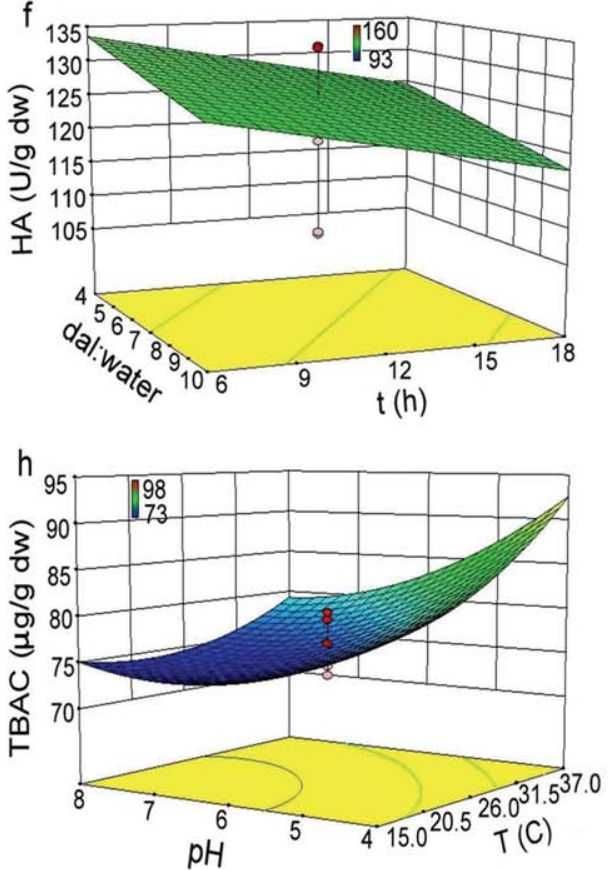

\section{Fermentation}

The levels of antinutrients under different fermentation conditions as per RSM design are shown in Table 3. The models for fermentation stage are significant with nonsignificant lack of fit (Table 7). The $R^{2}$ values show that independent variables explain more than $75 \%$ variation for each antinutrient. The adjusted and predicted $R^{2}$ values for models are in reasonable agreement, and adequate precision for models has ratio greater than 4 . The low values of $C V, S D$ and PRESS for models show the models are fit for prediction.

Fermentation time and temperature were significant for minimizing the levels of all the antinutrients, except total biogenic amines. Although salt concentration had a reducing effect on total biogenic amines content, it did not affect the other antinutrients. Reduced equations for significant 

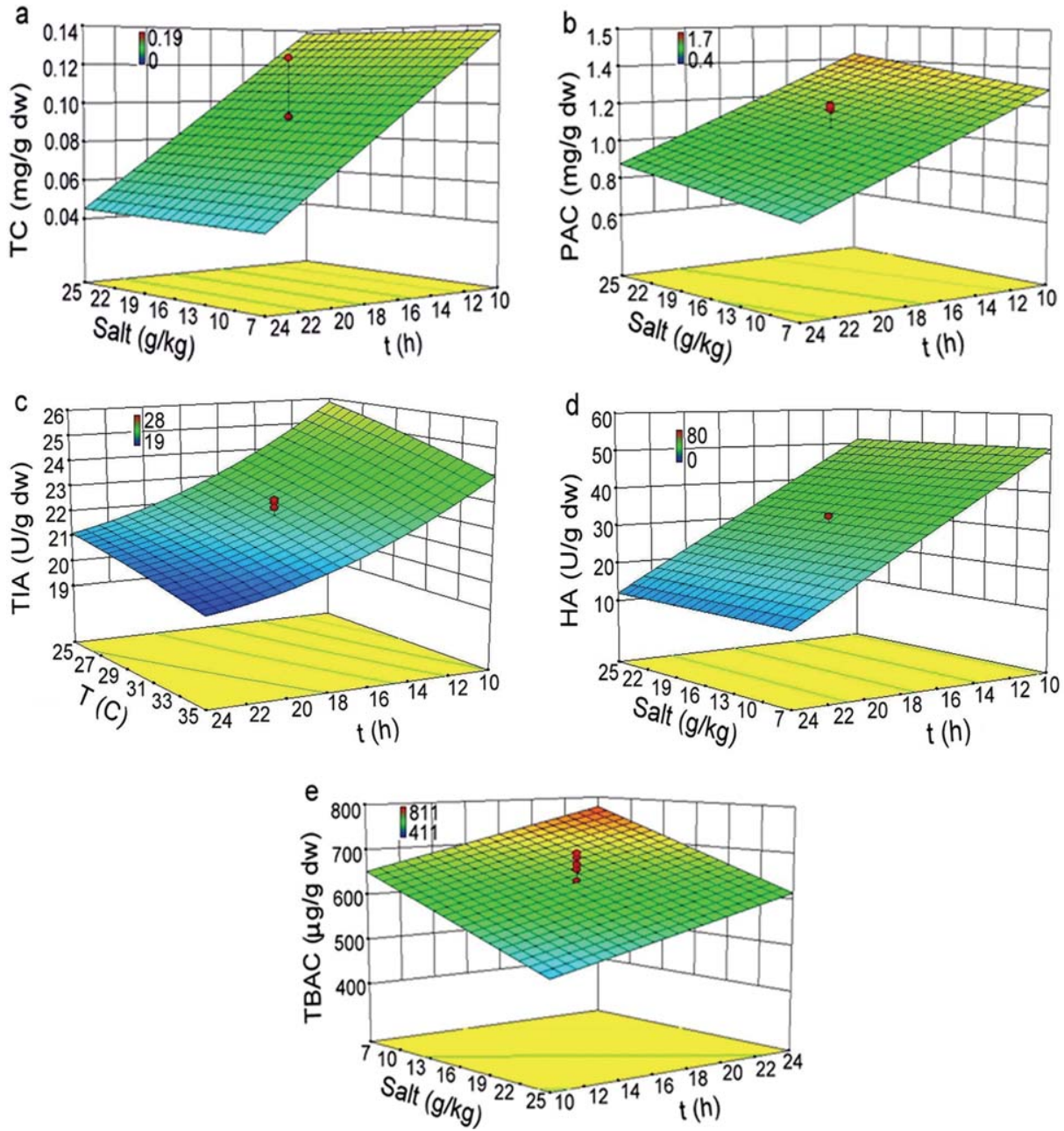

FIG. 2. RESPONSE SURFACE $3 D$ PLOTS SHOWING THE INFLUENCE OF SALT CONCENTRATION, FERMENTATION TIME ( $t$ ) AND FERMENTATION TEMPERATURE $(T)$ ON ANTINUTRIENTS: INFLUENCE OF ADDED SALT AND $t$ ON TANNINS CONTENT (TC; a), PHYTIC ACID CONTENT (PAC; b), HEMAGGLUTINATING ACTIVITY (HA; d) AND TOTAL BIOGENIC AMINES CONTENT (TBAC, e) WHEN $T$ WAS KEPT CONSTANT AT 30C; INFLUENCE OF $T$ AND T ON TRYPSIN INHIBITOR ACTIVITY (TIA; c) WHEN ADDED SALT CONCENTRATION WAS KEPT CONSTANT AT $16 \mathrm{~g} / \mathrm{kg}$
$(P<0.05)$ linear terms for tannins content, phytic acid content, hemagglutinating activity and total biogenic amines content and quadratic terms for trypsin inhibitor activity were:

Tannins content $(\mathrm{mg} / \mathrm{g})=0.091-0.047 B-0.026 C$

Phytic acid content $(\mathrm{mg} / \mathrm{g})=1.085-0.277 B-0.185 C$

Hemagglutinating activity $(\mathrm{U} / \mathrm{g})=31.667-19.615 B-$ $12.426 C$

Total biogenic amines content $(\mu \mathrm{g} / \mathrm{g})=633.707-$ $81.090 A+62.260 B+30.337 C$

Trypsin inhibitor activity $(\mathrm{U} / \mathrm{g})=22.189-2.183 B-$ $0.751 C+0.630 B^{2}$

where $A$ was salt concentration; $B$ was fermentation time and $C$ was fermentation temperature.

With an increase in fermentation time from 10 to $24 \mathrm{~h}$ at $30 \mathrm{C}$, there was a linear reduction in tannins content $(0.04 \mathrm{mg} /$ g; Fig. 2a), phytic acid content $(0.7 \mathrm{mg} / \mathrm{g}$; Fig. 2b) and hemagglutinating activity (12 U/g; Fig. 2d). The predicted reduction of tannins content, phytic acid content and hemagglutinating activity was 56, 59 and 70\%, respectively, over unfermented mixed batter. When the salt concentration was fixed at
$16 \mathrm{~g} / \mathrm{kg}$, the minimum level of trypsin inhibitor activity (20 U/g; Fig. 2c) obtained at $24 \mathrm{~h}$ and 35C was due to its reduction by $39 \%$. The reduction of tannins in fermented batter signifies an extensive leaching during soaking and fermentation. Reduction in the level of tannins content might be due to polyphenol oxidase, produced by the microorganisms during fermentation (Reddy and Pierson 1994). The reduction of phytic acid content in the present study might be due to phytase activity of the fermenting microorganisms. Microbial fermentation can enhance bioavailability of dietary minerals by hydrolyzing phytic acid using their own phytase (Kumar et al. 2010). The reduction of hemagglutinating activity and trypsin inhibitor activity during fermentation has been reported earlier (Reddy and Pierson 1994; Holzapfel 2002).

The minimum level of total biogenic amines content (493 $\mu \mathrm{g} / \mathrm{g}$; Fig. 2e) was obtained at $10 \mathrm{~h}$ of fermentation when the salt concentration was increased to $25 \mathrm{~g} / \mathrm{kg}$, keeping fermentation temperature fixed at 30C.

The total biogenic amines content in fermented batter was almost three times more than that of mixed 
unfermented batter. This is because biogenic amines are formed by several microbial groups possessing decarboxylase activity during fermentation (Holzapfel 2002). Interestingly, in this study, a reduction in the level of total biogenic amines content was observed when the salt concentration was increased. Similar results were reported by Chander et al. (1989). The high concentration of salt leads to reduced cell yield and progressively disturbs the membrane-located microbial decarboxylase (Sumner et al. 1990).

\section{Optimized Fermentation Condition}

Although maximum reduction of antinutrients was observed at salt concentration of $25 \mathrm{~g} / \mathrm{kg}$, presence of such a high concentration of salt in finished products may affect organoleptic quality and not be acceptable to consumers. Thus, based on the desirability $(P<0.01)$ of various concentrations of salts, $16 \mathrm{~g}$ salt $/ \mathrm{kg}$ was selected. Similarly, although fermentation for $24 \mathrm{~h}$ gave maximum reduction of antinutrients, a period of $19 \mathrm{~h}$ was selected as the duration of fermentation. Therefore, the optimized fermentation condition was $16 \mathrm{~g} / \mathrm{kg}$ added salt at $35 \mathrm{C}$ for $19 \mathrm{~h}$. The predicted reduction of antinutrient levels is shown in Table 6. The experimental values agreed with the predicted ones.

\section{Steaming}

Regression coefficient data show models for steaming stage to be significant with nonsignificant lack of fit (Table 7). This implies that model terms could explain more than $98 \%$ of variation for reduction in antinutrient levels. These results indicate a high precision in predicting reduction in antinutrient levels. The adjusted and predicted $R^{2}$, adequate precision, $C V, S D$ and PRESS indicate adequate model discrimination.

Phytic acid content, trypsin inhibitor activity and total biogenic amines content reduced $(P<0.05)$ with the increase in steaming time. The reduced significant linear equations were:

Phytic acid content $(\mathrm{mg} / \mathrm{g})=0.410-0.288 A+0.236 A^{2}$ $+0.271 A^{3}-0.302 A^{4}$

Trypsin inhibitor activity $(\mathrm{U} / \mathrm{g})=17.650-1.047 \mathrm{~A}+$ $2.303+0.427 \mathrm{~A}^{3}-2.013 \mathrm{~A}^{4}$

Total biogenic amines content $(\mu \mathrm{g} / \mathrm{g})=639.577-$ $11.357 A-0.329 A^{2}+7.877 A^{3}$

where $A$ was steaming time.

When steaming time was raised to $20 \mathrm{~min}$, the levels of antinutrients reduced sharply (Table 4 ). The phytic acid content increased initially (run numbers: 7 to 3), however after 12.5 min (run numbers: 3 to 1 ) it decreased. Such a time-dependent change in phytic acid content during heating at 100C was observed earlier (Kim and Kim 1998). The decrease in phytic acid content can be due to its degradation by heat or formation of insoluble complexes between

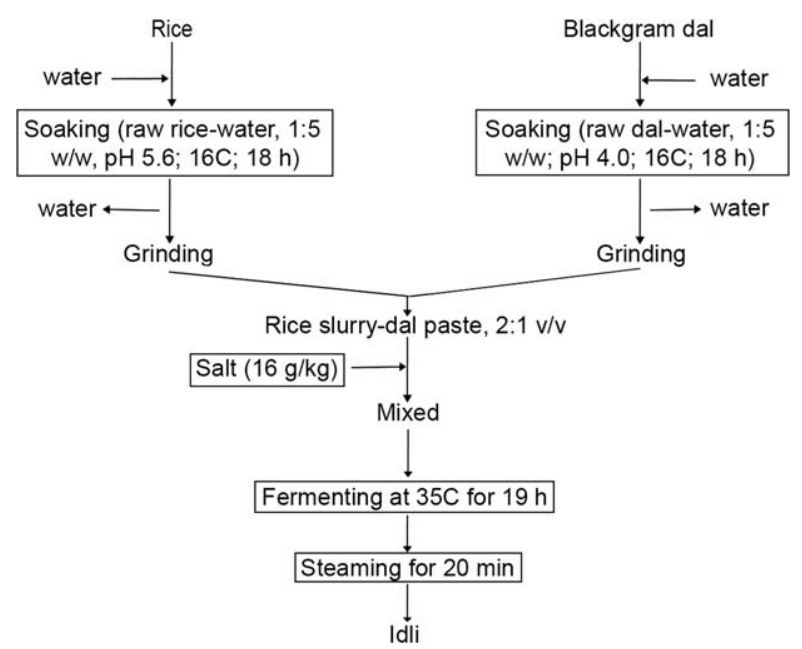

FIG. 3. THE OVERALL OPTIMIZED AND EXPERIMENTALLY VALIDATED PROCESS CONDITIONS (WITHIN BOXES) OF IDLI-MAKING

phytate and proteins or minerals (Vijayakumari et al. 2007). During steaming, destruction of disulfide bonds, splitting of covalent bonds or hydrolysis of peptide bonds might have caused a reduction of trypsin inhibitor activity (Adams 1991). Since hemagglutinin is heat-sensitive, it reduced below the limit of detection during the steaming process. Total biogenic amines content reduced by $6.6 \%$, indicating steaming for $20 \mathrm{~min}$ is effective in removal of total biogenic amines content in the final product.

\section{Optimized Steaming Condition}

The predicted optimum time for steaming condition was 20 min. Reduction of antinutrient levels is shown in Table 6. Although there was an increase $(P<0.05)$ of total biogenic amines content during optimum fermentation condition, it reduced during steaming. Since the levels of total biogenic amines content $(636 \mu \mathrm{g} / \mathrm{g})$ in optimally produced idli are below the hazardous level of $1000 \mu \mathrm{g} / \mathrm{g}$ food (Silla Santos 1996), such a product is safely consumable.

Optimum production processes of idli with minimum level of antinutrients are shown in Fig. 3 and Table 6. Traditionally prepared idli contained $0.06 \mathrm{mg}, 0.7 \mathrm{mg}, 24 \mathrm{U}, 0 \mathrm{U}$ and $641 \mu \mathrm{g}$ of tannins, phytic acid, trypsin inhibitor activity, hemagglutinating activity and total biogenic amines, respectively, per gram dry weight of idli.

\section{Microbiological Study}

The counts of total aerobic mesophilic bacteria, lactic acid bacteria and yeasts were studied for both unfermented and fermented mixed batters under different experimental conditions (Table 3). The microbial content, initial pH (5.6) and titratable acidity $(0.03 \%)$ values of unfermented batter changed rapidly during the course of fermentation. 
With the increase in salt concentration, lactic acid bacterial and yeast counts reduced compared to total aerobic mesophilic bacterial count, indicating a low salt concentration favored the growth of lactic acid bacteria and yeasts (Table 3). Again, with the increase in fermentation time not only lactic acid bacterial and yeast counts but total aerobic mesophilic bacterial count also increased. At the onset of fermentation, the population of total aerobic mesophilic bacteria exceeded $(P<0.05)$ that of lactic acid bacteria $(1: 0.9)$, indicating the presence of a relatively large number of nonlactic acid bacteria. However, after fermentation, the situation reversed (1:1.2), indicating that lactic acid bacteria had outgrown (dominated) other (nonlactic acid) bacteria, which in combination with the demonstrated acidification - is a sign of successful lactic acid fermentation. A similar situation was observed during lactic acid fermentation of various food grains (Agarwal et al. 2000; Shimelis and Rakshit 2008). Reduction $(P<0.05)$ in $\mathrm{pH}$ of batter by lactic acid bacteria allowed yeasts to grow (Soni and Sandhu 1991).

\section{Sensory Analysis}

Idli of good quality should have a slight sour aroma and a spongy texture with honey-comb crumb interior. Idli prepared from batter (having $16 \mathrm{~g} / \mathrm{kg}$ salt) fermented under optimized conditions of $35 \mathrm{C}$ for $19 \mathrm{~h}$ scored maximum (94), reaching to the "excellent" quality level (Table 5).

\section{CONCLUSION}

Traditionally, idli is prepared by soaking blackgram dal and rice (dal/rice:water, 1:4-1:10) at room temperature (15-37C) overnight (6-18 h), grinding soaked dal and rice, mixing of those (1:1-1:4) along with common salt (7-25 g/kg), fermenting at room temperature $(25-35 \mathrm{C})$ for $6-24 \mathrm{~h}$ and steaming for 15-20 min. Whereas the optimum condition for idli preparation in the present study was soaking of dal and rice (dal/rice:water, $1: \sim 5$ ) at $16 \mathrm{C}$ for $18 \mathrm{~h}$ followed by grinding and mixing of those (1:2) along with $16 \mathrm{~g} / \mathrm{kg}$ common salt, fermenting at $35 \mathrm{C}$ for $19 \mathrm{~h}$ and steaming for $20 \mathrm{~min}$.

In case of traditionally prepared idli, there was reduction of tannins content, phytic acid content, trypsin inhibitor activity and hemagglutinating activity by 70, 74, 40 and $100 \%$, respectively, over raw ingredients. On the other hand, the optimization of processing treatments caused a reduction $(P<0.05)$ of their respective levels by $100,89,58$ and $100 \%$. There was no difference $(P<0.05)$ between the two processes with respect to changes in total biogenic amines content. Thus, by deploying CCRD, idli-making was optimized towards a minimum level of antinutrients without affecting the organoleptic attributes of the product. The outcome of this study has a potential of scaling up idli production.

\section{ACKNOWLEDGMENT}

This work was supported by grant, F.3-4/2013 (SAP-II), from the University Grants Commission, New Delhi, India.

\section{REFERENCES}

ADAMS, J.B. 1991. Review: Enzyme inactivation during heat processing of food-stuffs. Int. J. Food Sci. Technol. 26, 1-20.

AGARWAL, R., RATI, E.R., VIJAYENDRA, S.V.N., VARADARAJ, M.C., PRASAD, M.S. and NAND, K. 2000. Flavour profile of idli batter prepared from defined microbial starter cultures. World J. Microbiol. Biotechnol. 16, 687-690.

AIDOO, K.E., NOUT, M.J.R. and SARKAR, P.K. 2006. Occurrence and function of yeasts in Asian indigenous fermented foods. FEMS Yeast Res. 6, 30-39.

AOAC. 1990. Official Methods of Analysis, 15th ed., Assoc. of Official Analytical Chemists, Arlington, VA.

ASHENAFI, M. and BUSSE, M. 1991. Microflora of soak water during tempeh production from various beans. J. Appl. Bacteriol. 70, 334-338.

BOYE, J., ZARE, F. and PLETCH, A. 2010. Pulse proteins: Processing, characterization, functional properties and applications in food and feed. Food Res. Int. 43, 414-431.

CHANDER, H., BATISH, V.K., BABU, S. and SINGH, R.S. 1989. Factors affecting amine production by a selected strain of Lactobacillus bulgaricus. J. Food Sci. 54, 940-942.

GREWAL, A. and JOOD, S. 2006. Effect of processing treatments on nutritional and antinutritional contents of green gram. J. Food Biochem. 30, 535-546.

HEMALATHA, S., PLATEL, K. and SRINIVASAN, K. 2007. Zinc and iron contents and their bioaccessibility in cereals and pulses consumed in India. Food Chem. 102, 1328-1336.

HETTIARACHCHY, N.S. and SRI KANTHA, S.S. 1981. Trypsin inhibitor and phytohaemagglutinin contents in the seeds of six legumes commonly consumed in Sri Lanka. J. Nat. Sci. Counc. Sri Lanka 9, 269-272.

HOLZAPFEL, W.H. 2002. Appropriate starter culture technologies for small-scale fermentation in developing countries. Int. J. Food Microbiol. 75, 197-212.

JOOSTEN, H.M.L.J. and VAN BOEKEL, M.A.J.S. 1988. Conditions allowing the formation of biogenic amines in cheese. 4. A study of the kinetics of histamine formation in an infected Gouda cheese. Neth. Milk Dairy J. 42, 3-24.

KAKADE, M.L., SIMONS, N. and LIENER, I.E. 1969. An evaluation of natural vs. synthetic substrates for measuring the antitryptic activity of soybean samples. Cereal Chem. 46, 518-526.

KALPANADEVI, V. and MOHAN, V.R. 2013. Effect of processing on antinutrients and in vitro protein digestibility of the underutilized legume, Vigna unguiculata (L.) Walp subsp. unguiculata. LWT - Food Sci. Technol. 51, 455-461. KHANDELWAL, S., UDIPI, S.A. and GHUGRE, P. 2010. Polyphenols and tannins in Indian pulses: Effect of soaking, germination and pressure cooking. Food Res. Int. 43, 526-530. 
KIM, Y.M. and KIM, Y.W. 1998. Changes of enzyme activity, trypsin inhibitor, tannin and phytic acid during heat treatment of soybean. Korean J. Food Sci. Technol. 30, 1012-1017.

KUMAR, V., SINHA, A.K., MAKKAR, H.P.S. and BECKER, K. 2010. Dietary roles of phytate and phytase in human nutrition - A review. Food Chem. 120, 945-959.

LIENER, I.E. and HILL, E.G. 1953. The effect of heat treatment on the nutritive value and hemagglutinating activity of soybean oil meal. J. Nutr. 49, 609-620.

MONTGOMERY, D.C. 2005. Design and Analysis of Experiments, 6th Ed., John Wiley \& Sons, New York.

NOUT, M.J.R., SARKAR, P.K. and BEUCHAT, L.R. 2007. Indigenous fermented foods. In Food Microbiology: Fundamentals and Frontiers, 3rd ed. (M.P. Doyle and L.R. Beuchat, eds.) pp. 817-835, ASM Press, Washington, D.C.

PRICE, M.L., VAN SCOYOC, S. and BUTLER, L.G. 1978. A critical evaluation of the vanillin reaction as an assay for tannin in sorghum grain. J. Agric. Food Chem. 26, 1214-1218.

RAKSHIT, M., SHARMA, A., SAHA, J. and SARKAR, P.K. 2015. Optimization of soaking condition of blackgram to minimize flatogenic sugar content in blackgram-based products. LWT - Food Sci. Technol. 63, 814-820.

REDDY, N.R. and PIERSON, M.D. 1994. Reduction in antinutritional and toxic components in plant foods by fermentation. Food Res. Int. 27, 281-290.
SAHARAN, K., KHETARPAUL, N. and BISHNOI, S. 2002. Antinutrients and protein digestibility of faba bean and rice bean as affected by soaking, dehulling and germination. J. Food Sci. Technol. 39, 418-422.

SHIMELIS, E.A. and RAKSHIT, S.K. 2008. Influence of natural and controlled fermentations on $\alpha$-galactosides, antinutrients and protein digestibility of beans (Phaseolus vulgaris L.). Int. J. Food Sci. Technol. 43, 658-665.

SILLA SANTOS, M.H. 1996. Biogenic amines: Their importance in foods. Int. J. Food Microbiol. 29, 213-231.

SONI, S.K. and SANDHU, D.K. 1991. Role of yeast domination in Indian idli batter fermentation. World J. Microbiol. Biotechnol. 7, 505-507.

SUMNER, S.S., SPECKHARD, H.W., SOMERS, E.B. and TAYLOR, S.L. 1990. Factors controlling histamine production in Swiss cheese inoculated with Lactobacillus buchneri. J. Dairy Sci. 73, 3050-3058.

VIJAYAKUMARI, K., PUGALENTHI, M. and VADIVEL, V. 2007. Effect of soaking and hydrothermal processing methods on the levels of antinutrients and in vitro protein digestibility of Bauhinia purpurea L. seeds. Food Chem. 103, 968-975.

WHEELER, E.L. and FERREL, R.E. 1971. A method of phytic acid determination in wheat and wheat fractions. Cereal Chem. 48, 312-320.

YEH, C.Y., LIN, S.J. and HWANG, D.F. 2006. Biogenic amines, histamine and label of dressed fried fish meat products in Taiwan. Food Control 17, 423-428. 